\title{
Incident Type 2 Diabetes Risk is Influenced by Obesity and Diabetes in Social Contacts: a Social Network Analysis
}

\author{
Sridharan Raghavan, MD, PhD ${ }^{1,2,3}$, Mark C. Pachucki, PhD', Yuchiao Chang, PhD', \\ Bianca Porneala, $M S^{7}$, Caroline S. Fox, MD', Josée Dupuis, PhD ${ }^{6,7}$, and James B. Meigs, $M D^{7}$
}

\begin{abstract}
'Division of General Internal Medicine, Department of Medicine, Harvard Medical School and Massachusetts General Hospital, Boston, MA, USA; ${ }^{2}$ Department of Veterans Affairs Medical Center, Eastern Colorado Health Care System, Denver, CO, USA; ${ }^{3}$ Division of General Internal Medicine, Department of Medicine, University of Colorado School of Medicine, Denver, CO, USA; ${ }^{2}$ Department of Sociology, Computational Social Science Institute, University of Massachusetts, Amherst, MA, USA; ${ }^{5}$ Laboratory for Metabolic and Population Health, National Heart, Lung, and Blood Institute, Framingham, MA, USA; ${ }^{6}$ Department of Biostatistics, Boston University School of Public Health, Boston, MA, USA; ${ }^{7}$ The National Heart, Lung, and Blood Institute's Framingham Heart Study, Framingham, MA, USA.
\end{abstract}

BACKGROUND: Obesity and diabetes family history are the two strongest risk factors for type 2 diabetes (T2D). Prior work shows that an individual's obesity risk is associated with obesity in social contacts, but whether T2D risk follows similar patterns is unknown.

OBJECTIVE: We aimed to estimate the relationship between obesity or diabetes in an individual's social contacts and his/ her T2D risk. We hypothesized that obesity and diabetes in social contacts would increase an individual's T2D risk.

DESIGN: This was a retrospective analysis of the community-based Framingham Offspring Study (FOS).

PARTICIPANTS: FOS participants with T2D status, height and weight, and at least one social contact were eligible for this study ( $n=4797$ at Exam 1). Participants' interpersonal ties, cardiometabolic and demographic variables were available at eight exams from 1971 to 2008, and a T2D additive polygenic risk score was measured at the fifth exam.

MAIN MEASURES: Primary exposures were T2D (fasting glucose $\geq 7 \mathrm{mmol} / \mathrm{L}$ or taking diabetes medications) and obesity status (BMI $\geq 30 \mathrm{~kg} / \mathrm{m}^{2}$ ) of social contacts at a prior exam. Primary outcome was incident T2D in participants.

KEY RESULTS: Incident T2D was associated with having a social contact with diabetes (OR 1.32, $p=0.004$ ) or with obesity (OR 1.21, $p=0.004$ ). In stratified analyses, incident T2D was associated with diabetes in siblings (OR 1.64, $p=$ 0.001 ) and obesity in spouses (OR 1.54, $p=0.0004)$. The associations between diabetes and obesity in social contacts and an individual's incident diabetes risk were stronger in individuals with a high diabetes genetic risk score.

CONCLUSIONS: T2D and obesity in social contacts, particularly siblings and spouses, were associated with an individual's risk of incident diabetes even after accounting for parental T2D history. Assessing risk factors in an individual's siblings and spouses can inform T2D risk; furthermore, social network based lifestyle interventions involving spouses and siblings might be a novel T2D prevention approach.

Electronic supplementary material The online version of this article (doi:10.1007/s11606-016-3723-1) contains supplementary material, which is available to authorized users.

Received October 8, 2015

Revised March 23, 2016

Accepted April 20, 2016

Published online May 4, 2016
KEY WORDS: type 2 diabetes; social network; obesity; family history; epidemiology.

J Gen Intern Med 31(10):1127-33

DOI: $10.1007 / \mathrm{s} 11606-016-3723-1$

(c) Society of General Internal Medicine 2016

\section{INTRODUCTION}

The last decade has seen rapid progress in the identification of how social relationships contribute to a variety of weight-related health behaviors, ${ }^{1-6}$ body weight, ${ }^{7-10}$ and chronic disease status. ${ }^{11,12}$ This research shows that attributes or behaviors of the people in one's social network can serve as important determinants of one's own health. However, one's social network often includes family members, which can make it difficult to discern the relative impact of shared behaviors and shared genetics for familial common chronic diseases.

Type 2 diabetes (T2D) is produced by a convergence of behavioral and familial risk factors. Rates of T2D are rapidly growing worldwide, and a number of risk factors contribute to incident diabetes, most prominently obesity, family history, and age. ${ }^{13,14}$ Cross-sectional research on T2D and impaired glucose metabolism has suggested that diabetes clusters within social networks. ${ }^{15-17}$ However, mechanisms responsible for this phenomenon have been hard to discern, given the scarcity of informative longitudinal data on social relationships and T2D. Furthermore, relative contributions of shared behaviors and shared genetics to familial aggregation of T2D are poorly understood and likely vary across individuals and families. How social relationships and genetic background may independently and prospectively affect diabetes development could have implications for T2D pathophysiology and offer insight into social levers for prevention.

The aim of this study was to examine the association of an individual's risk of incident T2D with obesity or T2D in his/ her related and unrelated social contacts, and to determine if underlying genetic risk of diabetes modified that association. To establish a basis for studying possible social influences on 
incident T2D risk, we first examined whether prevalent cases of T2D cluster across social ties in the Framingham Heart Study (FHS). Second, we performed the primary analysis to examine whether an individual's risk of incident T2D, the primary outcome, was associated with diabetes and obesity in his/her social contacts. The data in FHS allowed interrogation of whether obesity or diabetes in specific types of social contacts - siblings, spouses, friends, neighbors - was particularly influential on an individual's risk of incident T2D, and we examined these associations in stratified analyses. We hypothesized that being socially connected to individuals with T2D or obesity would be associated with an increased risk of incident T2D; that diabetes and obesity in relatives would confer greater incident T2D risk than in unrelated social contacts; and that risk across social ties would be relatively higher in individuals with greater T2D genetic risk.

\section{METHODS}

\section{Study Population}

The Framingham Heart Study (FHS) is a well-described prospective cohort study begun in 1948. ${ }^{18}$ Measurements of fasting glucose, diabetes medications, height, weight, selfreported parental history of T2D, educational attainment, and social contacts were obtained through the Database of Genotypes and Phenotypes (dbGaP) from the National Center for Biotechnology Information. We focus here on only the offspring of the original cohort members and their spouses (Framingham Offspring Study, FOS) and track their T2D status and diabetes risk factors over eight examination periods occurring every 3 to 5 years between 1971 and 2008 .

Previous investigators coded administrative data describing social connections between cohort participants to produce a database of exam-specific social ties. ${ }^{7,19}$ The terminology of social network analysis describes an "ego" (the individual in whom the outcome is being assessed), an "alter" (a cohort member whom an ego reported as a social contact), and "network size" (the number of alters connected to each ego). Of the 5124 individuals enrolled in the FOS at baseline, the public data set accessible to researchers through $\mathrm{dbGaP}$ includes 4801 participants, due to privacy restrictions. We included egos with T2D status, height and weight, and at least one alter with available T2D status, height and weight from at least one exam cycle ( $n=4797$ at Exam 1). In addition, we restricted the analysis to spouse, friend, sibling, or close neighbor (same-address or next-door neighbors only) social ties, as these are relatively intimate ties where some type of social-behavioral influence might reasonably be expected to occur. In some cases, both members of a spousal or sibling pair were participants in the FOS; thus, in these cases, individuals could be captured as both egos and alters in the data set. The proportion of participants with network information was relatively stable and over $90 \%$ across all eight exams (online Supplemental Table S1). Between Exam 1 and Exam 8, there was some participant attrition due to mortality and non-response. Those who did not drop out early were on average younger, leaner, and more frequently had parental diabetes history than those who dropped out early (see Online-only Supplement). All FHS and FOS participants provided informed consent to their participation, and the Boston University Medical Campus and Partners Institutional Review Boards approved this study.

\section{Outcomes, Exposures, Covariates}

The primary outcome was incident T2D, defined as fasting glucose value greater than or equal to $7 \mathrm{mmol} / \mathrm{L}$, or taking diabetes medications, a definition that has been used previously in the FOS and that has been shown to be over $98 \%$ accurate in diagnosing T2D in the FOS. ${ }^{20}$ There were a total of 692 incident T2D cases over mean follow-up of 25 years. BMI in egos and alters was calculated as weight (kilograms) divided by the height (meters) squared. The two primary exposures of interest were alter T2D status, defined using the same criteria as ego T2D, and alter obesity, defined as a BMI greater than or equal to $30 \mathrm{~kg} / \mathrm{m}^{2}$. Parental history of T2D was a dichotomous variable based on ego self-report of whether either parent had T2D. ${ }^{21}$ Educational attainment was available in a subgroup of participants and took values from 1 (fourth grade or less) to 8 (post-graduate school). For our analyses, we dichotomized educational attainment at less than completing high school versus completing high school or greater.

\section{Genetic Risk}

Genotyping, quality control, and calculation of a T2D additive genetic risk score (GRS) in the FHS and FOS have been previously described. ${ }^{22}$ Briefly, the GRS was calculated as the weighted sum of the number of risk alleles at each of 62 loci associated with T2D for which index or proxy single nucleotide polymorphisms (SNPs) were available in Offspring Study genetic data, weighted by the effect size for association with diabetes. ${ }^{23,24}$ For stratified analyses of high vs. low GRS, we dichotomized the study population at the median (66.9) weighted GRS. As only a subset of participants had genetic data and educational data available, we compared those with or without genetic data and those with or without educational data for substantial differences in baseline characteristics in the Onlineonly Supplement. Briefly, those with genetic data or with educational data were, on average, younger and leaner and more often had parental diabetes history than those without genetic data or without educational data (see online Supplemental Tables S2 and S3).

\section{Statistical Analysis}

To account for known T2D risk factors, all models were adjusted for ego age, sex, and parental history of T2D, as well as for an indicator of exam cycle to account for secular trends in diabetes and obesity incidence. To account for possible effects associated with degree of social connectedness, we also adjusted all models for ego network size. As we were 
also interested in T2D genetic risk, we adjusted for the diabetes GRS in the subset of participants for whom those data were available.

Our goal was to estimate relationships between ego T2D and (a) alter T2D and (b) alter obesity. To do so, we used a series of three progressively restrictive dyadic network models in a generalized estimating equation (GEE) framework with the logit link function. ${ }^{7,19,25}$ All models accounted for correlation due to repeated measures by clustering on ego, and we used the independence correlation structure, which has previously been shown to yield the most unbiased estimates based on ego-alter correlations in this data set. ${ }^{19}$

The first model (Model 1), which examined clustering of T2D cases in the FHS social network, estimated the relationship between prevalent ego T2D (at a given time $t$ ) as the outcome, and contemporaneous alter T2D and obesity (at the same time $t$ ) as the key exposures, adjusting for ego covariates. The second model (Model 2), which represents our primary analytic model, estimated associations between alter traits (obesity and T2D) at the prior exam and incident T2D in non-diabetic egos; that is, the primary exposures in Model 2 were alter obesity and alter $\mathrm{T} 2 \mathrm{D}$ at the prior exam, and the primary outcome was incident ego T2D, adjusting for ego covariates at the prior exam.

To address the hypothesis that associations between incident ego T2D and alter traits were stronger across ties between related ego-alter dyads than unrelated dyads, we repeated the analyses stratifying by the type of relationship; we first stratified by related versus unrelated ties, and then secondarily explored each of the unrelated ties that were represented in our data set-sibling, spouse, friend, neighbor. Similarly, to address the hypothesis that ego T2D genetic risk would modify associations between alter traits and incident ego diabetes, we repeated the analyses stratifying participants into high and low 62 SNP diabetes GRS categories at the median GRS for the study population.

Finally, in secondary analyses, we introduce a third model (Model 3) that aimed to identify evidence of social influence of alters on an ego's T2D risk. Unlike Model 2, Model 3 includes time-varying obesity exposures to account for correlations between ego and alter traits and to assess whether accounting for a change in ego's obesity attenuated the association between ego and alter's diabetes status. We adapted this last model from the cross-lagged panel model used to discern peer associations in obesity status by Christakis and Fowler. $^{19}$

The modeling strategy is described in greater detail in the Online-only Supplement. As our primary hypothesis tested whether two exposures, alter obesity and alter T2D, would be associated with incident ego diabetes, we applied a significance threshold of $p<0.025$. Network size was calculated using the package sna in R; GEE models were run in SAS 9.3 (SAS Institute, Inc, Cary, NC) and confirmed using the package geeglm in R.

\section{RESULTS}

\section{Study Participants}

At exam 1 of the Offspring Study, 4797 study participants were on average overweight (BMI $25.4 \pm 4.4 \mathrm{~kg} / \mathrm{m}^{2}$ ); there were 136 cases of T2D at baseline, and over the course of the study, there were a total of 692 incident cases of diabetes (Table 1 and online Supplemental Table S1). Across eight exams, the number of dyads in the data set ranged from 14,434 (exam 1) to 9309 (exam 8); ties between siblings (41-46\%) and between spouses (23-24\%) were most common (Table 1 and online Supplemental Table S1).

\section{Alter Obesity and Diabetes are Associated with Incident Ego Diabetes}

Preliminary data inspection confirmed the association between ego and alter obesity described in prior work ${ }^{7}$ (online Supplemental Table S5). The first analysis step showed clustering of prevalent ego T2D with alter diabetes [OR $1.38(1.18,1.61)$, $p<0.0001$, online Supplemental Table S6, Model 1] and with alter obesity [OR $1.17(1.04,1.33), p=0.01$, online Supplemental Table S7, Model 1; see Online-only Supplement]. We then tested the primary hypothesis that alter traits predicted incident ego T2D. To this end, we examined whether alter obesity or diabetes at the prior exam was associated with incident T2D in non-diabetic egos (Model 2 described above). Separate analyses revealed that both alter T2D [OR 1.32 (1.09, $1.59), p=0.004$ ] and alter obesity [OR $1.21(1.06,1.37), p=$ $0.004]$ were significantly associated with incident ego diabetes, even after adjusting for ego BMI (Fig. 1a; online Supplemental Tables S6-S7, Model 2).

The family-based structure of the FHS allowed us to test whether a related versus unrelated relationship type between

Table 1. Study Participant Characteristics at Exam 1

\begin{tabular}{lll}
\hline \hline Characteristic & $\boldsymbol{N}(\%)$ & Mean (SD) \\
\hline Ego Sex & & \\
$\quad$ Female & $2466(51)$ & - \\
Ego Age & 4797 & $36.2(10.5)$ \\
Ego Education Attainment & $712(65)$ & - \\
$\quad$ < High School & $1316(35)$ & - \\
$\quad \geq$ High School & & \\
Ego T2D Status & $4661(97)$ & - \\
$\quad$ No T2D & $136(3)$ & - \\
$\quad$ T2D & $4153(87)$ & - \\
Ego T2D Parental History & $644(13)$ & - \\
$\quad$ No T2D history & 4797 & $25.4(4.41)$ \\
$\quad$ T2D history & $4158(87)$ & - \\
Ego BMI (continuous) & $639(13)$ & - \\
Ego Obesity Status & 4797 & $4.2(2.9)$ \\
$\quad$ Not obese (<30 kg/m $\left.{ }^{2}\right)$ & 2498 & $66.9(5.1)$ \\
$\quad$ Obese ( $\left.\geq 30 \mathrm{~kg} / \mathrm{m}^{2}\right)$ & & \\
Ego Network size & $5910(41)$ & \\
Ego 62 SNP Genetic Risk Score & $2090(14)$ \\
Count of Ego-Alter dyads & $3125(22)$ & \\
$\quad$ Siblings & $3309(23)$ & \\
$\quad$ Friends &
\end{tabular}


A

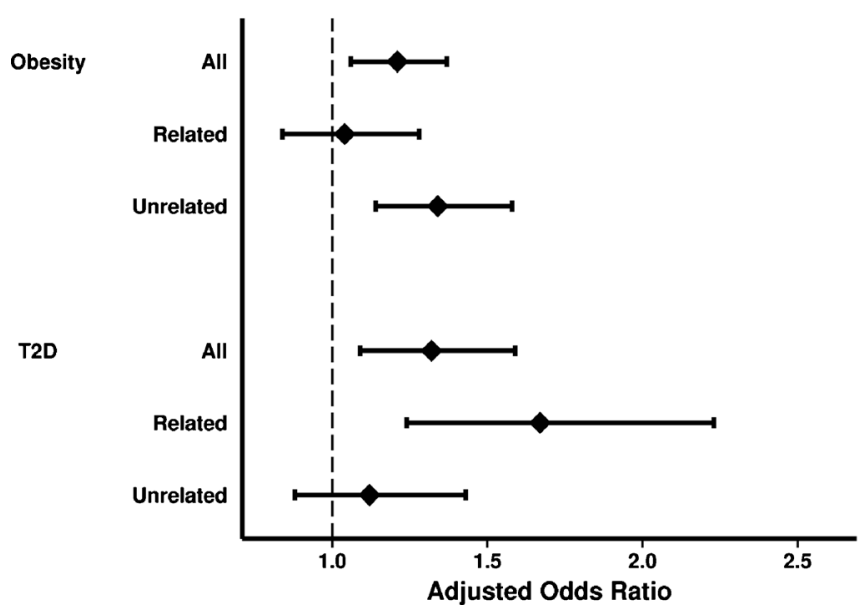

B

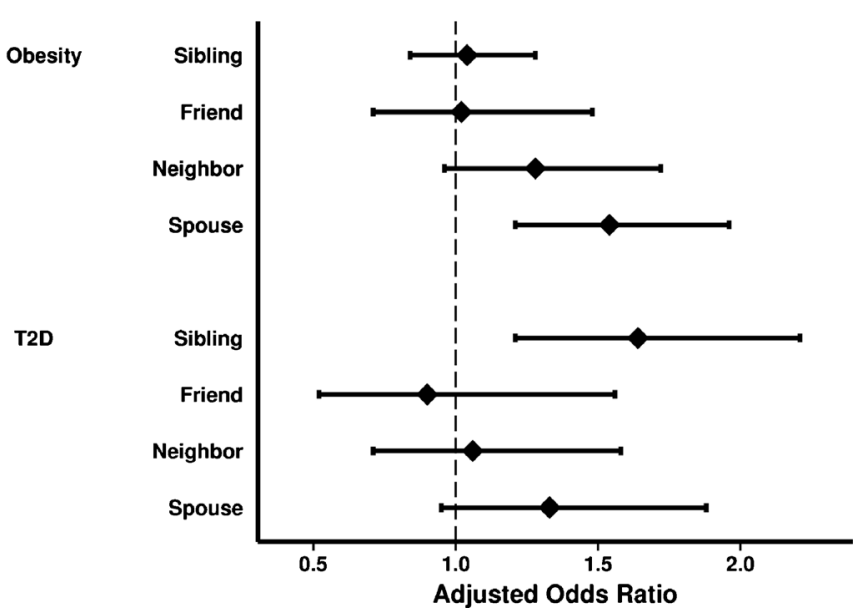

Figure 1. Associations of alter obesity or T2D with incident ego T2D, stratified by social contact type. Panel a shows the association of alter obesity or alter diabetes at the prior exam with incident ego T2D across all, related, and unrelated ties after adjusting for ego age, sex, parental history of T2D, BMI, network size, and exam cycle. Panel b shows the association of prior alter obesity or prior alter diabetes with incident ego T2D across specific social tie types. Prior alter obesity and diabetes were associated with incident ego T2D, and unrelated alter obesity and related alter diabetes were associated with incident ego T2D.

ego and alter modified the association between alter traits and incident ego T2D. Stratifying by ties between related versus unrelated individuals, we found that prior obesity in unrelated but not related alters was associated with incident ego diabetes (Fig. 1a; online Supplemental Table S8, Model 2). Stratifying further by type of social tie (spousal versus non-spousal), spousal obesity specifically was associated with a $54 \%$ increase in relative odds of incident ego T2D (Fig. 1b). Conversely, T2D at the prior exam in related (specifically siblings) but not unrelated alters was associated with incident ego diabetes, even after adjusting for parental history of T2D [OR $1.12(0.88,1.43), p=0.36$ across unrelated ties; OR 1.67 (1.24, 2.23), $p=0.0007$ across related ties; Fig. 1a and b; online Supplemental Table S9, Model 2).

To demonstrate more clearly how easily ascertained alter traits compare to traditional T2D risk factors, we repeated our analyses, but with dichotomous ego and alter obesity variables rather than continuous BMI. While an ego's prior obesity and parental history of diabetes were the strongest predictors of incident ego T2D, identifying an alter with diabetes or obesity at the prior exam increased an ego's relative odds of incident T2D by $32 \%$ or $25 \%$, respectively, even after adjusting for ego obesity and parental history of diabetes (Table 2). Stratifying by type of social contact even more dramatically showed that having a related alter with diabetes increased an ego's relative odds of incident T2D by $55 \%$, even after accounting for parental diabetes history, and that being connected to an obese unrelated alter increased an ego's relative odds of incident T2D by $35 \%$ (Table 2).

\section{Alter Influences on Incident Ego Diabetes Vary According to Ego Diabetes Additive Genetic Risk}

Common genetic variants can be added to estimate genetic risk for $\mathrm{T}_{2} \mathrm{D}^{22,24}$ and $\mathrm{T} 2 \mathrm{D}$ genetic risk scores may interact with environmental exposures on diabetes risk. ${ }^{27,28}$ Accordingly, we tested whether an ego's T2D GRS modified associations of alter traits with incident ego diabetes. To this end, we

Table 2. Association of Ego and Alter Risk Factors for T2D with Incident Ego T2D, Stratified by Social Contact Type

\begin{tabular}{|c|c|c|c|c|c|c|}
\hline \multirow[b]{2}{*}{ Characteristic } & \multicolumn{2}{|l|}{ All ties } & \multicolumn{2}{|l|}{ Related ties } & \multicolumn{2}{|l|}{ Unrelated ties } \\
\hline & OR (95\% CI) & $p$ value & OR (95 \% CI) & $p$ value & OR (95 \% CI) & $p$ value \\
\hline Ego age & $1.06(1.05,1.07)$ & $<0.0001$ & $1.06(1.05,1.08)$ & $<0.0001$ & $1.06(1.05,1.07)$ & $<0.0001$ \\
\hline Ego sex & $1.45(1.17,1.80)$ & 0.0006 & $1.52(1.15,2.00)$ & 0.003 & $1.43(1.13,1.82)$ & 0.003 \\
\hline Ego family history & $2.61(2.07,3.29)$ & $<0.0001$ & $2.37(1.75,3.21)$ & $<0.0001$ & $2.78(2.16,3.59)$ & $<0.0001$ \\
\hline Ego obesity $\left(\mathrm{t}_{-1}\right)$ & $3.75(3.02,4.64)$ & $<0.0001$ & $4.05(3.08,5.33)$ & $<0.0001$ & $3.57(2.81,4.52)$ & $<0.0001$ \\
\hline Alter obesity $\left(\mathrm{t}_{-1}\right)$ & $1.25(1.11,1.42)$ & 0.0004 & $1.11(0.90,1.37)$ & 0.31 & $1.35(1.14,1.59)$ & 0.0004 \\
\hline Alter diabetes $\left(\mathrm{t}_{-1}\right)$ & $1.32(1.10,1.58)$ & 0.003 & $1.55(1.17,2.06)$ & 0.003 & $1.18(0.93,1.49)$ & 0.18 \\
\hline
\end{tabular}

Model: Outcome is incident ego T2D at a given exam (time t); primary exposures are alter obesity at the prior exam (time $t_{-1}$ ) and alter T2D at the prior exam (time $t_{-1}$ ), adjusting for ego age, sex, parental T2D history, ego obesity at the prior exam (time $\left.t_{-1}\right)$, and ego network size

Abbreviations: OR Odds Ratio; $95 \%$ CI $95 \%$ Confidence Interval

$p$ values are from multivariable generalized estimating equations applied to a binary outcome (T2D) 
Table 3. Association of Ego and Alter Risk Factors for T2D with Incident Ego T2D, Stratified by Ego T2D Genetic Risk Score

\begin{tabular}{|c|c|c|c|c|}
\hline \multirow[b]{2}{*}{ Characteristic } & \multicolumn{2}{|c|}{ Low genetic risk score" } & \multicolumn{2}{|c|}{ High genetic risk score ${ }^{*}$} \\
\hline & OR (95\% CI) & $p$ value & OR (95\% CI) & $p$ value \\
\hline Ego age & $1.07(1.04,1.10)$ & $<0.0001$ & $1.05(1.03,1.07)$ & $<0.0001$ \\
\hline Ego sex & $1.65(1.01,2.68)$ & 0.04 & $1.30(0.93,1.82)$ & 0.13 \\
\hline Ego parental history & $2.79(1.69,4.58)$ & $<0.0001$ & $2.62(1.83,3.76)$ & $<0.0001$ \\
\hline Ego obesity $\left(t_{-1}\right)$ & $4.34(2.71,6.95)$ & $<0.0001$ & $4.41(3.08,6.32)$ & $<0.0001$ \\
\hline Alter obesity $\left(\mathrm{t}_{1}\right)$ & $0.96(0.71,1.30)$ & 0.79 & $1.34(1.10,1.64)$ & 0.004 \\
\hline Alter diabetes $\left(\mathrm{t}_{-1}\right)$ & $1.22(0.76,1.96)$ & 0.40 & $1.35(1.02,1.77)$ & 0.03 \\
\hline
\end{tabular}

Model: Outcome is incident ego T2D at a given exam (time t); primary exposures are alter obesity at the prior exam (time $t_{-1}$ ) and alter T2D at the prior exam (time $\left.t_{-1}\right)$, adjusting for ego age, sex, parental T2D history, ego obesity at the prior exam (time $\left.t_{-1}\right)$, and ego network size Abbreviations: OR Odds Ratio; $95 \%$ CI $95 \%$ Confidence Interval

$p$ values are from multivariable generalized estimating equations applied to a binary outcome (T2D)

*Low T2D genetic risk score was defined as $<66.9$ (the median GRS), and high genetic risk score was defined as $\geq 66.9$

first examined whether the associations between ego T2D and alter traits could be explained by ego diabetes GRS in the approximately $50 \%$ of study participants for whom we had genetic data. Across all ties, incident ego T2D remained significantly associated with prior alter diabetes, but not prior alter obesity, after adjusting for ego T2D GRS (Fig. 2a, online Supplemental Tables S10 and S11). However, in analysis stratified by related versus unrelated alter and adjusted for ego T2D GRS, we observed that obesity in an unrelated alter increased the relative odds of incident ego T2D by $31 \%$ (Fig. 2a, online Supplemental Table S11).

We also stratified the data according to low versus high ego T2D GRS to test whether the association between alter traits and incident ego diabetes differed across strata of ego diabetes genetic risk. We found that prior alter obesity was significantly associated with incident T2D in egos with a high T2D GRS (OR 1.34), but not those with a low GRS (OR 0.96; $p$ value for interaction $<0.0001$ ); similarly, prior alter diabetes was significantly associated with incident T2D only in egos with a high diabetes GRS ( $p$ value for interaction 0.01; Fig. $2 b$, Table 3).

\section{Secondary Analyses}

Our primary analyses addressed whether alter traits provide information that helps predict an ego's risk of developing T2D. We performed secondary analyses to address whether being connected to an alter with obesity or diabetes was associated with an ego's risk of developing T2D regardless of time. To this end, we employed a model that adjusted for prior and contemporaneous ego and alter BMI (Model 3 in METHODS). We found that incident ego T2D remained associated with prior alter diabetes [OR $1.34(1.09,1.64), p=$ 0.005; online Supplemental Table S6, Model 3], but that incident ego T2D was no longer associated with alter obesity [OR $1.15(0.94,1.40), p=0.17$; online Supplemental Table S7, Model 3]. When stratifying by relationship type, diabetes at the prior exam in related alters increased the relative odds of incident ego T2D by $70 \%$, even after
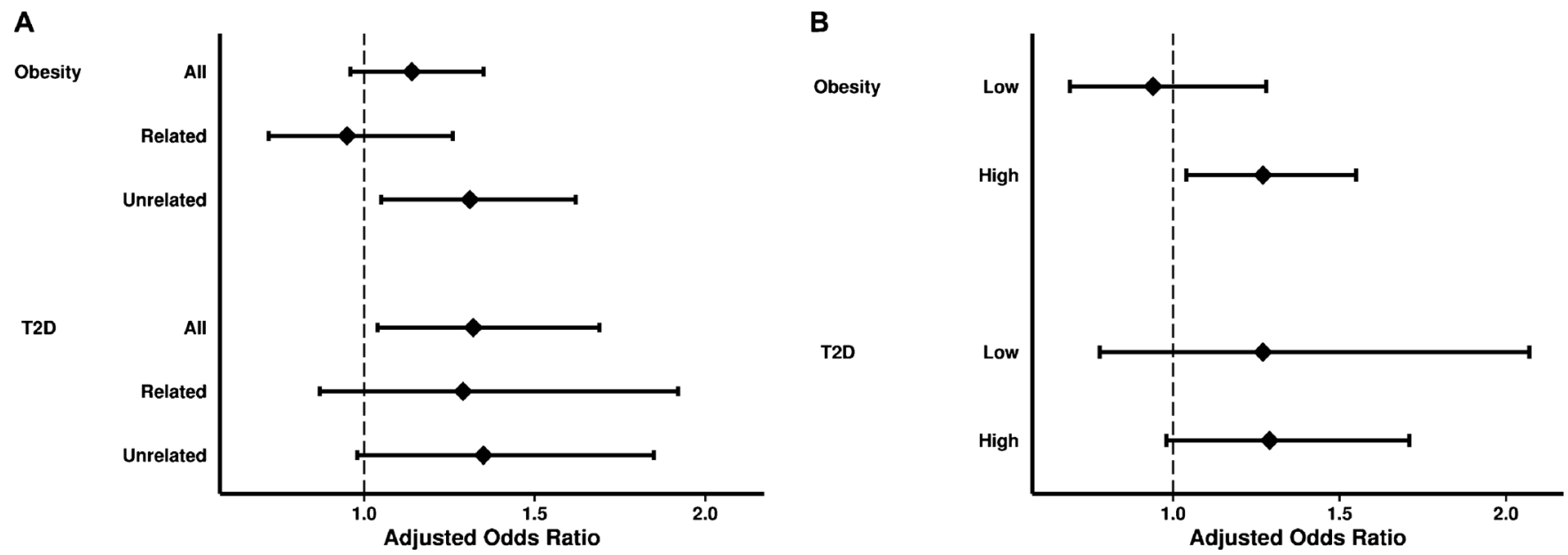

Figure 2. Associations of alter obesity or T2D with incident ego T2D, adjusting for or stratifying by ego T2D genetic risk. Panel a shows the association between prior alter obesity or prior alter diabetes and incident ego T2D across all, related, and unrelated ties after adjusting for ego T2D genetic risk score (GRS). Panel b shows the association between prior alter obesity or prior alter diabetes and incident ego T2D, stratified according to low (below the median) and high (median and higher) ego T2D GRS. Prior unrelated alter obesity remained a significant predictor of incident ego T2D, and prior alter diabetes remained significantly associated with incident ego T2D, even after adjusting for ego diabetes GRS. Association between alter obesity and alter T2D and incident ego T2D was stronger for egos with a high GRS than those with a low GRS ( $p$ value for interaction $<0.0001$ for alter obesity and 0.01 for alter T2D). 
adjusting for prior and contemporaneous ego and alter BMI (online Supplemental Table S9, Model 3). In contrast, neither T2D nor obesity in unrelated alters were associated with incident ego T2D in this model (online Supplemental Tables S8 and S9, Model 3).

Finally, our primary analyses do not capture socioeconomic attributes known to cluster within a social network and known to be important and potentially modifiable risk factors for T2D..$^{29,30}$ Including ego educational attainment did not significantly change the associations between alter diabetes and incident ego T2D, but did attenuate the association between alter obesity and incident ego diabetes, particularly across unrelated ties (online Supplemental material, online Supplemental Table S12).

\section{DISCUSSION}

In this study of longitudinal social network data from the Framingham Heart Study, we found that obesity and T2D in a person's social contacts was associated with their own risk of developing T2D. This extends prior work demonstrating peer associations in BMI and insulin resistance. Specifically, we observed that obesity but not diabetes in a spouse and diabetes but not obesity in a sibling were strongly associated with an individual's risk of incident diabetes. Furthermore, we found that alter diabetes remained associated with incident ego T2D even after adjusting for ego GRS and parental history, indicating that the association between alter and ego diabetes was unlikely due solely to shared obesity or shared genetic/familial risk. Additionally, we found that a person's T2D genetic risk modified the effect of social contact obesity and diabetes on the risk of developing T2D.

While social network analyses can be threatened by unmeasured homophily, that is, a tendency towards similarity in traits between socially connected individuals, our analytic models account for observed homophily in diabetes and obesity. We hypothesize that the ego-alter associations we observed are mediated by shared health behaviors, particularly diet and exercise. This comports with and extends cross-sectional research by Henning and colleagues, who found weight-related attitudes and behaviors to be implicated in peer group associations with insulin resistance. ${ }^{15}$ A practical implication for care provision is that systematically adding information about spouses, siblings, friends, and household members to conventional family and social history elements in primary care encounters could enhance identification of patients at particularly high risk of developing diabetes who already harbor risk factors such as obesity or parental diabetes history. Furthermore, prevention interventions designed to target a high-risk person and his or her social network members jointly may prove efficacious. ${ }^{10,30,31}$ Given the difficulty of behavior change for T2D prevention, our work suggests that social and familial context might be important considerations in the ongoing search for effective and practical diabetes and obesity prevention strategies. Finally, if common genetic variant tests for T2D are useful, they could be combined with social contact trait information to identify people most likely to benefit from social network-based prevention strategies.

Our study has some limitations. The FHS social network data set was derived from administrative data that was collected to maximize study follow-up. As such, the data did not necessarily identify all —or even the most important — members of a person's social network. However, as siblings and spouses comprise the largest proportion of social contacts in our data, we could parse important familial and non-familial influences on T2D risk. Another limitation is participant attrition; however, because those who did not drop out early were on average younger and leaner than those who did drop out, it is possible that our findings underestimate the magnitude of associated obesity and T2D among social contacts. Next, prior work applying the GEE framework to correlated network data to estimate social influence has been performed in the context of reversible outcomes. In contrast, we have treated diabetes, once acquired, as an irreversible state. To accommodate this modeling assumption, we used an approach that limited analysis of incident cases to T2D acquisition across consecutive exam cycles. Despite this limitation, we detected significant effects that lay the groundwork for more detailed investigation of peer effects on incident T2D. Finally, our analyses have not included all known risk factors for T2D, particularly diet, physical activity, blood pressure, lipid measurements, and socioeconomic status in all participants, which may be potential mediators of an association between alter metabolic traits and ego diabetes. Prior work has shown that while many of these variables are significantly associated with incident T2D, each of these variables individually contribute little to discrimination of incident $\mathrm{T} 2 \mathrm{D},{ }^{32}$ and many of these variables (diet, physical activity, and socioeconomic status) were unavailable longitudinally in the FHS.

Our work provides novel, quantitative evidence of association between social relationships and incident T2D within a social network. By combining strong predictors of T2D with new social network information and genetic risk information, we have extended our understanding of the relative contributions of environmental and biological factors that give rise to diabetes. The association between an individual's risk of incident diabetes and spousal obesity supports investigation of lifestyle interventions targeted at households or spousal dyads. At minimum, the associations observed between alter traits and incident ego T2D suggest that ascertainment of risk factors of close social contacts might provide valuable data for risk assessment and preventive counseling of patients.

Author Contributions: SR, MCP, and JBM conceived and designed the study; SR, MCP, CSF, JD, and JBM contributed to data acquisition; SR, MCP, YC, BP, JD, and JBM contributed to data analysis; all authors contributed to data interpretation; all authors contributed to drafting and critical revisions of the manuscript as well as final approval of the version submitted for review and publication. SR and JBM had full 
access to all the data in the study and take responsibility for the integrity of the data and the accuracy of the data analysis.

Corresponding Author: James B. Meigs, MD; Division of General Internal Medicine, Department of MedicineHarvard Medical School and Massachusetts General Hospital, Boston, MA, USA (e-mail: jmeigs@partners.org).

\section{Compliance with Ethical Standards:}

Funding: SR is supported by NIH National Research Service Award T32HP10251 and the Ryoichi Sasakawa Fellowship Fund. JBM and JD are supported by NIH RO1DK78616, and JBM is also supported by K24 DK080140. We gratefully acknowledge the FHS study participants and staff for their contributions. This work was partially supported by the National Heart, Lung and Blood Institute's Framingham Heart Study (Contract No. NO1-HC-25195) and its contract with Affymetrix, Inc for genotyping services (Contract No. NO2-HL-6-4278).

Prior Presentations: Elements of this manuscript were presented (1) orally at the Society of General Internal Medicine Annual Meeting on 24 April 2015 in Toronto, Canada and (2) as a poster at the American Diabetes Association Scientific Sessions on 7 June 2015 in Boston, MA.

Conflict of Interest: The authors have no conflicts of interest to disclose pertaining to this work.

\section{REFERENCES}

1. Leroux JS, Moore S, Richard L, Gauvin L. Physical inactivity mediates the association between the perceived exercising behavior of social network members and obesity: a cross-sectional study. PLoS One. 2012;7(10), e46558.

2. Larson N, Wall M, Story M, Neumark-Sztainer D. Home/family, peer, school, and neighborhood correlates of obesity in adolescents. Obesity. 2013;21(9):1858-69.

3. Simpkins SD, Schaefer DR, Price CD, Vest AE. Adolescent friendships, BMI, and physical activity: untangling selection and influence through longitudinal social network analysis. J Res Adolesc. 2013;23(3):537-49.

4. Robinson E, Thomas J, Aveyard P, Higgs S. What everyone else is eating: a systematic review and meta-analysis of the effect of informational eating norms on eating behavior. J Acad Nutr Diet. 2014;114(3):414-29.

5. Conklin AI, Forouhi NG, Surtees P, Khaw K-T, Wareham NJ, Monsivais P. Social relationships and healthful dietary behaviour: evidence from over50s in the EPIC cohort. UK Soc Sci Med. 2014;100:167-75.

6. Pachucki MA, Jacques PF, Christakis NA. Social network concordance in food choice among spouses, friends, and siblings. Am J Public Health. 2011;101(11):2170-7.

7. Christakis NA, Fowler JH. The spread of obesity in a large social network over 32 years. N Engl J Med. 2007;357(4):370-9.

8. Schaefer DR, Simpkins SD. Using social network analysis to clarify the role of obesity in selection of adolescent friends. Am J Public Health. 2014; 104(7): 1223-29.

9. Wang ML, Pbert L, Lemon SC. Influence of family, friend and coworker social support and social undermining on weight gain prevention among adults. Obesity. 2014;22(9):1973-80.

10. Pachucki MC, Goodman E. Social relationships and obesity: benefits of incorporating a lifecourse perspective. Curr Obes Rep. 2015; In press.

11. Keating NL, O'Malley AJ, Murabito JM, Smith KP, Christakis NA. Minimal social network effects evident in cancer screening behavior. Cancer. 2011;117(13):3045-52.
12. Loucks EB, Sullivan LM, D'Agostino RB Sr, Larson MG, Berkman LF, Benjamin EJ. Social networks and inflammatory markers in the Framingham Heart Study. J Biosoc Sci. 2006;38(6):835-42.

13. Danaei G, Finucane MM, Lu Y, Singh GM, et al. National, regional, and global trends in fasting plasma glucose and diabetes prevalence since 1980: systematic analysis of health examination surveys and epidemiological studies with 370 country-years and $2 \cdot 7$ million participants. Lancet. 2011;378:31-40.

14. Wilson PWF, Meigs JB, Sullivan L, Fox CS, Nathan DM, D'Agostino RB. Prediction of incident diabetes mellitus in middle-aged adults: the Framingham Offspring Study. Arch Intern Med. 2007; 167:1068-74.

15. Henning $\mathbf{C H}$, Zarnekow N, Hedtrich J, Stark S, Turk K, Laudes M. Identification of direct and indirect social network effects in the pathophysiology of insulin resistance in obese human subjects. PLoS One. 2014;9(4), e93860.

16. Hilding A, Shen C, Ostenson CG. Social network and development of prediabetes and T2D in middle-aged Swedish women and men. Diabetes Res Clin Pract. 2015;107(1): 166-77.

17. Hempler NF, Ekholm O, Willaing I. Differences in social relations between persons with T2D and the general population. Scand J Public Health. 2013;41(4):340-3.

18. Feinleib M, Kannel WB, Garrison RJ, McNamara PM, Castelli WP. The Framingham offspring study. Design and preliminary data. Prev Med. 1975;4(4):518-25.

19. Christakis NA, Fowler JH. Social contagion theory: examining dynamic social networks and human behavior. Stat Med. 2013;32(4):556-77.

20. Meigs JB, Cupples LA, Wilson PW. Parental transmission of type 2 diabetes: the Framingham offspring study. Diabetes. 2000;49:220107.

21. Murabito JM, Nam BH, D'Agostino RB Sr, Lloyd-Jones DM, O'Donnell CJ, Wilson PW. Accuracy of offspring reports of parental cardiovascular disease history: the Framingham offspring study. Ann Intern Med. 2004; 140(6):434-40.

22. Vassy JL, Hivert M-F, Porneala B, et al. Polygenic T2D prediction at the limit of common variant detection. Diabetes. 2014;63:2172-82.

23. Morris AP, Voight BF, Teslovich TM, et al. Large-scale association analysis provides insights into the genetic architecture and pathophysiology of T2D. Nat Genet. 2012;44(9):981-90.

24. Meigs JB, Shrader P, Sullivan LM, et al. Genotype score in addition to common risk factors for prediction of T2D. N Engl J Med. 2008;359:2208-19.

25. O'Malley AJ. The analysis of social network data: an exciting frontier for statisticians. Stat Med. 2013;32(4):539-55.

26. Langenberg C, Sharp SJ, Franks PW, et al. Gene-lifestyle interaction and T2D: the EPIC interact case-cohort study. PLoS Med. 2014;11(5), e1001647.

27. Qi Q, Chu AY, Kang JH, Huang J, et al. Fried food consumption, genetic risk, and body mass index: gene-diet interaction analysis in three US cohort studies. BMJ. 2014;348:g1610.

28. Maty SC, Everson-Rose SA, Haan MN, Raghunathan TE, Kaplan GA. Education, income, occupation, and the 34-year incidence (196599) of T2D in the Alameda County Study. Int J Epidemiol. 2005;34(6): 1274-81.

29. Sacerdote C, Ricceri F, Rolandsson O, et al. Lower educational level is a predictor of incident T2D in European countries: the EPIC-InterAct study. Int J Epidemiol. 2012;41(4):1162-73.

30. Valente TW. Network interventions. Science. 2012;337(6090):49-53.

31. Voils CI, Coffman CJ, Yancy WS Jr, et al. A randomized controlled trial to evaluate the effectiveness of CouPLES: a spouse-assisted lifestyle change intervention to improve low-density lipoprotein cholesterol. Prev Med. 2013;56(1):46-52.

32. Wilson PW, Meigs JB, Sullivan L, et al. Prediction of incident diabetes mellitus in middle-aged adults: The Framingham offspring study. Arch Intern Med. 2007; 167:1068-74. 